\title{
Principal management concepts in Greek public sector: Part II - Management by Objectives
}

\author{
Ioannis PAPALAZAROU, \\ Hellenic Open University, School of Social Sciences, Parodos Aristotelous 18, 26335, Patra, Greece, \\ janellsis@yahoo.gr \\ Giannis T. TSOULFAS*, \\ Hellenic Open University, School of Social Sciences, Parodos Aristotelous 18, 26335, Patra, Greece, \\ tsoulfas.ioannis@ac.eap.gr \\ * Corresponding author
}

\begin{abstract}
The introduction of specific and measurable objectives in the public sector is a practice that seeks to increase the efficiency of public services. This is done with their clear and acceptable identification, both at an individual and organisational level, their measurement with specific indicators and the benchmarking of the results. Goal setting is the essential tool for the specification and implementation of planning for various time horizons. As a detailed guide of organisational priorities, it constitutes the procedure of the determination of clear goals and objectives, deriving from the upper hierarchical level of each organization and their subsequent diffusion to the lower levels in the form of specific actions. This paper is the second and last part of a study about the application of principal management concepts in Greek public sector since year 2004 when Law 3230/2004 was introduced. In this part the experience of the implementation of "Management by Objectives" in Greek public services is analysed. Towards this end, the views and opinions of experts from the public sector are analyzed with the use of interviews and questionnaires. The aggregated results from both parts of the study are presented and discussed by the end of this paper.
\end{abstract}

Keywords: Management by Objectives; public administration; Total Quality Management; Greece

JEL Classification: D73, L30

\section{Introduction}

In recent years, with the advancement of technology, the advances in eGovernment and the various changes required by the impositions of European regulations, the modernisation of the public administration can no longer be considered a choice but a necessary requirement for any modern state organisation (Black et al., 2001; Pollitt and Bouckaert, 2011). The rapid socioeconomic developments have led the institutions of the European Union to 
actions aimed at successfully meeting the needs and expectations of partalis, on which the attention is now concentrated, by improving the quality of services offered, because it is the quality of public services that makes public administration reliable in the public eye (Staes et al., 2010).

In order to assist public administration in achieving quality, it is necessary to design and apply simplifying procedures, improve the interaction between the state and its partalis and take advantage of the potentials of modern technology. The objective of any modern public administration, therefore, should be the creation of services characterised by flexibility, simplified procedures and reduced red tape (Rammata, 2011). The term "Public management" first appeared in the 1970's, expressing more of an institutional development than simply something abstract or theoretic. Since then, the transition from public administration to public management has been rapid; however, even today the distinction between the two terms is not clearly visible. The key feature of this concept is that the main role of public administration is offering services and not governing, i.e. not exercising power through authoritarian means and repressive controls. According to this approach managerial actions should be focused on the satisfaction of public needs and evaluated only in qualitative terms. In this context, the main orientation of public management is the application of generally accepted principles of scientific management, with an emphasis on specialisation, the diffusion of work, the hierarchical structure of authority, the use of financial and human resources, the implementation of modern leadership concepts, staff motivation and performance measurement.

In order for quality to prevail in public administration procedures and contribute to its overall improvement, a transition from the traditional bureaucratic model of public management is necessary. The principles and requirements of public management have significant differences from those expressed by the model of bureaucracy which has been followed for years in public administrations worldwide. In contrast with the bureaucratic system of organisation, public management can contribute in finding practical and sustainable solutions, but the fact is that most government departments and agencies are still applying bureaucratic procedures, not being, thereby, able to shake off its pathogenic characteristics (Esmark, 2016). The distinctive differences between bureaucracy and public management, as identified by Borins (1995), are presented in Table 1. 
Table 1. Differences between Bureaucracy and Public management

\begin{tabular}{cc}
\hline BUREAUCRACY & PUBLIC MANAGEMENT \\
\hline Long-term planning & Short-term planning \\
Static Targeting & Dynamic targeting \\
Avoiding errors & Pursuing Success \\
importance & importance \\
Economic resources are of minor financial & Economic resources are of major final \\
Hierarchical and rigid structure & Austere structure \\
Limited delegation of decision making & Maximum delegation of decision making \\
Focus on the types & Focus on results \\
\hline
\end{tabular}

The study of quality and the use of quality tools and systems began in the private sector after WWII and followed an evolutionary path: first, it was only associated with compliance to technical specifications and standards, next the element of "fitness for use" was introduced, afterward the focus was transferred to the alignment with customer requirements to be today on the achievement of maximum customer satisfaction. The evolution of the content of quality in the private sector was, much later, followed by a similar path in the public sector (Staes and Thijs, 2005).

The current paper is focused on the application of $\mathrm{MBO}$ and is structured as follows. In section 2 the concepts of evaluation and assessment are discussed followed by the analysis of MBO. In section 3 the introduction of MBO in the Greek public sector is analysed and the research methodology is outlined. The major findings are discussed in section 4 . Section 5 provides the integrated concluding remarks of the study on the implementation of the CAF and MBO in the Greek public sector.

\section{The role of evaluation and assessment in the modernisation of public administration: Management By Objectives}

The concept of evaluation was introduced through the application of quality assurance systems; however, it was not always associated with improvement because the basic goal was initially to comply with specific standards (e.g. ISO 9000 series). Its connection with the concept of improvement came through the introduction of TQM, with a number of assessment and improvement tools (e.g. EFQM) which are nowadays available to public and private organisations. With the term "improvement" is meant any change of the 
performance of an organisation which has beneficial effects on the satisfaction of the partali-customer. Quality improvement may refer to production costs reductions, increased productivity, improvement in employee morale, etc (Psomas and Jaca, 2016).

The evaluation of an organisation can take the form of either a) selfassessment, in which the organisation evaluates itself or b) hetero-evaluation where the organisation evaluates or is evaluated by another organisation. Selfassessment provides a tool of self-diagnosis and a basis for improvements as it contributes towards meeting the basic requirement of achieving quality improvement for an organisation, which is that a quality system should be in accordance with specific procedures, in other words that the quality system reflects, in real terms, the actual behavior of employees in their workplace.

However, the biggest drawback of self-assessment is that it requires objective measurements which can accurately translate the quality practices followed by the organisation in relation to the quality standard chosen. But in order to perform these measurements trained staff with the necessary knowledge and experience is needed, the availability of which is not always certain. The presence of this kind of employees is vital since these people are responsible for the analysis of the results and will design the required improvement measures and actions (Metaxas and Koulouriotis, 2014).

As far as how the evaluation of public organisations is being carried out, the process, compared to that of private firms, has significant differences. These derive from the difference in the nature, the structure and the goals of private enterprises in comparison with public ones. There have been many approaches in determining the differences between public and private organisations and their objectives. The primary goals of private enterprises in comparison with public agencies are different. For any private enterprise its main objective is efficiency, meaning maximum level of output for the given input. On the contrary, the goal of a public agency is equality and justice in the distribution of services.

Public agencies are different from private institutions because their main goals and objectives are not the same (Lee, 2011). The ultimate goal of private business is to maximise its profits, in its need to survive in the market in which it operates and from which it to raises its necessary funds. Differences also exist in the way resources are ensured and that, in consequence, has a different effect on how the organisation is managed. On the other hand, public services are a part of the government which plays a distinct role different from the one of a business in the market. Public bodies derive their power from the Law and their funds derive from the taxes with their commitment, and aim, being the provision of the service.

Having in mind all of the above, the lack of the profit factor which largely determines the effectiveness and sustainability of a private company, the nature 
of services provided by the public sector and the demand for equal and fair distribution of these services to all partalis make the evaluation of the performance of public services a very complex and complicated process (Rathgeb Smith, 2010).

Administration led by objectives achievement is defined as the process of identifying strategic objectives in higher hierarchical levels and diffusing them in the form of specific actions in lower hierarchical levels. More specifically, at the top of the administrative pyramid there are objectives, which are determined by the mission of the organisation and are specified by the leadership's strategy and vision. When deciding on goals, they are first defined in the upper administrative levels and then in the lower ones, therefore a "from top to bottom" approach is followed: doing so, the diversification increases, the range of responsibilities narrows, actions have an executive nature and goals are specified in detail. The opposite occurs in the goals realisation phase, where a "from bottom to top" approach is followed. At the bottom there are goals, which specify the objectives and the expected operational results. Here, sectional goals corresponding to specific duties are realised in order to achieve the set strategic objectives (Tsekos, 2007).

Reference to MBO dates back in 1954 when Peter Drucker, in his 1954 book "The Practice of Management", presented the concept the concept as the administrative organisation system that works efficiently when all individual functions are aligned with the overall strategic planning, as well as with the specialised operational objectives of each directorate or department (Rammata, 2011).

The essence of $\mathrm{MBO}$ is participative goal setting - when employees are involved in setting the objectives and choosing the courses of action to follow, they are more likely to fulfill their responsibilities. Also, an important part of the application is the measurement and comparison of performance results against the standards set.

In order for the effective implementation of the planned objectives the following elements should occur (Drucker, 1976):

- the vision and mission of the organisation should be clearly stated, with the strategic objectives being consistent with them.

- it is important that a relative degree of autonomy exists, in the sense that employees must be free to act and take initiatives in decisionmaking and their other various actions 
- the support of both political and administrative leadership is fundamental prerequisite in the correct implementation of MBO

- through goal setting clear goals should be set in order to achieve

- the desired results. During the decision on goals the agreement between employees and their superiors is desired

- all stakeholders must be involved in the decision-making process. This promotes co-perception throughout the organisation.

- a results feedback mechanism should exist, in order for the appropriate corrective measures to be taken, which must be based on the interactive relationship between superiors and subordinates.

- rewarding competent staff in case of attainment of the agreed objectives, by linking productivity with financial, and other, rewards, should also be considered.

\section{Insights on the application of $\mathrm{MBO}$ in the Greek public sector}

In the past the Greek state had turned into an instrument of serving the individual interests of the people in power, the guilds, the various professional groups a.o. resulting to the accumulation of the problems that, even to this day, hinder the development and modernisation of the public sector. Furthermore, the big influence of the political parties in public administration led, through shady recruitment systems in the limits of clientelism, to the existence of supernumerary and, in many cases of questionable capability, personnel. Additionally, public administration suffers from a lack of capable senior executives in key positions with management skills, since these are often filled by employees-party members or people from unions, which often do not have the required administrative capabilities or, in some cases, the will to follow organised plans or projects (Pappas and Assimakopoulou, 2012). One more pathogenesis of the Greek state is the misallocation of its employees. In most public agencies there is a surplus of workers in central and supportive departments and a lack of people in the "front line", i.e. in producing the actual services and dealing with customers face to face. Moreover, a lack in Modern Human Resource Management systems and procedures is also noted, such as recruitment processes, promotion, evaluation, rewards and incentives, which could also contribute to the development and improvement of employees and procedures (Rammata, 2011). 


\subsection{The establishment and dissemination of $M B O$ in Greek public services}

In regards to goal setting, the application has an annual span, while for the relative measurement indicators the frequency of their measurement is defined by a joint decision of the Ministry of Interior Affairs and each respective Ministry (Hellenic Republic, 2004). In addition, both the MBO system as well as the measurement indicators of efficiency and effectiveness are applied in all public sector services, public entities, banks which are considered public entities (or in which the major shareholder in the state) and at local authorities of first and second degree.

The implementation procedure of the MBO application and the logic behind it resembles an "administrative pyramid": at the top (highest point) of the administrative pyramid are objectives, which are determined by the mission of each organisation and specified by the leadership's strategy and vision. At the bottom (lowest point) are goals, which specify the expected operational results and administrative actions. MBO and the subsequent measuring of performance by specific indicators are done according to the following methodology.

In the beginning of November a notion is sent by the Directorate of Structure Organisation and Evaluation (DSOE) to the Ministers reminding them of the decision which must be taken concerning the objectives that their respective Ministry must set for the following year. Each Minister, then, determines the strategic objectives of the public services for the upcoming year and announces the relative (joint, with the Ministry of Interior Affairs) ministerial decision to all heads of the General Directorates of the respective Ministry, not later than December 20th. By this decision the priority of each objective, the results measuring indicators and the implementation timetable are determined.

Afterwards, each General Division defines and describes its mission (as stated in the description of its responsibilities), decides on its strategic objectives with the issue of the relative goal-setting decision, and sets goals of individual action towards the effective and efficient completion of the operational tasks within the set strategic objectives. This is done annually by issuing the relative regulations in months November and December (until the 20th of December at the latest).This specification of strategic objectives into operational goals and programs of action depends on the hierarchical structure of each organisation, nevertheless the individual operational objectives of the relevant sector must comply with the wider strategic purposes of the organisation, and aid to their longterm implementation.

Next, the set goals of each directorate are distributed in its departments and the responsibilities of each employee are defined. Employees must, on their 
part, determine how, in particular, they will promote these objectives in order for the strategy of the service to be implemented. To decide on each employee contribution to the cause, the head of each department co-operates with each of them separately in order to determine quantitative and qualitative targets according to their knowledge, competencies, skills and experience (Tsimboukidou et al., 2013).

In general, a very important point in the successful implementation of the MBO system is the consent and agreement of all staff members for the set targets as well as their commitment towards the implementation timetable. These must be secured before the beginning of the process.

The implementation process officially starts in January and extends until November. During this period it is monitored by the heads of the Directorates at regular intervals, which should be no longer than three months. Depending on the results, the Directorate can revise and redefine goals and their implementation plan accordingly. The Directorates, by collecting the necessary data, measure each activity with special indicators, but in order for these results to be useful they need to be reliable, measurable and comparable. For this reason, the Directorate should appoint two employees, with knowledge and experience in Management, in order to coordinate and "run" the MBO application effectively in collaboration with the DQE of their institution and the DQE of the Ministry of Interior Affairs.

It should be noted, at this point, that at each level of the goal setting procedure the Quality and Efficiency units of each relative Ministry are informed for the actions taken. In the beginning of December the application is concluded and the Quality and Efficiency unit of each Directorate drafts and submits a detailed report on its actions in regards to the $M B O$ application and sends it to the DQE of the Ministry it belongs to. Then, the DQE of each Ministry reports its results and submits them to the DQE of the Ministry of Interior Affairs. The latter agency drafts and submits, between January and late February, the annual report regarding the implementation of $\mathrm{MBO}$ by all Ministries and submits it to the Minister of Interior Affairs. The process is completed with the submission, by the Minister of Interior Affairs, to the Prime Minister, the President of the Parliament and the rest of the Ministers of a report that includes the results of the measurements as well as the recommendation of the Minister for the design and implementation of specific measures aimed at improving effectiveness and efficiency. Furthermore, towards a more effective implementation of the MBO system, workshops are, from time to time, organised to inform the heads of General Divisions, Directorates, Departments and the employees with relative, to the application, tasks (EKDDA, 2012). 


\subsection{The research methodology}

As stated in the first part of the study, the primary research was conducted via interviews as well as the use of questionnaires. Interviewees and respondents include high ranking public officials ( $\mathrm{HO} 1, \mathrm{HO} 2, \mathrm{HO} 3, \mathrm{HO} 4, \mathrm{HO} 5$ and $\mathrm{HO} 6$ ) with a specialisation on CAF and MBO matters and lower ranking public employees (LE1 and LE2) with experience in the implementation of the two tools. Further insights on the characteristics of the selected methodology are provided in the first part of the study.

\section{Findings regarding the use of the MBO in the Greek public sector}

The research on $\mathrm{MBO}$ use in the Greek public sector revealed the following findings, many of them being similar to those of the CAF.

Once again, as a major drawback in the introduction of the tool the absence of a general quality framework, regulating the function of the public services was mentioned. There seems to be no general administrative planning aiming on improvement based on quality processes. The existence of a total quality policy, as HO1, HO4 and LE1 mentioned, would be beneficial towards the correct introduction and implementation of the $\mathrm{MBO}$. $\mathrm{HO} 2$, furthermore, mentioned the absence of a definite clarification, by the leadership, of the mission and vision of each public body and their dissemination to all staff.

Directly connected with the above was the ascertainment that there was, at least in the beginning, no adequate preparation for the introduction of the MBO: officials and employees were not sufficiently trained in order to implement the tool properly as $\mathrm{HO} 2$ and $\mathrm{HO} 6$ noted. $\mathrm{HO} 1$ mentioned that although some seminars on MBO were organised by INEP there were not performed in collaboration with DSOE (which would add to their credibility), organised repeatedly, or were reached all services. Lately, however, the number of seminars organised relative to the implementation of MBO are increased (EKDDA, 2012).

In essence Law 3240/04 is not in effect, HO2, HO6 and LE2 mentioned. What became soon evident during this research was that since the Law was not implemented correctly this naturally effected one of its tools, the MBO. The results of the implementation of the application in the Greek public sector are considered to be limited, as $\mathrm{HO} 1$ mentioned. MBO was not implemented as described in the provision of the Law. Most of the employees with participation on MBO implementation procedures, noted that the application was usually conducted up to a point, usually only until the objectives setting stage, but was almost never 
concluded properly, i.e. by setting correct measuring indicators, gathering and analysing the results.

Numerous reasons were mentioned explaining the above:

The most important obstacle in MBO implementation was the reluctance of many departments to proceed with the procedure. Although obligatory, many offices did not establish decisions on their goals, others stated them but did not proceed to measure their actions towards achieving them or to create a results report, HO6 and LE2 mentioned.

What also became evident, especially between lower rank public employees, was that they were not convinced of the feasibility and functionality of the application, this way depreciating its value as an improvement tool. This, as $\mathrm{HO} 4$ noted, can probably be explained by their insufficient training and difficulty to fully understand the procedure. That contributed in the lack of commitment towards the application and their relative duties and may also explain, as HO2 mentioned, the lack of trust of lower rank employees towards their leaders.

Furthermore, the deficient introduction of MBO caused suspicions among public employees, $\mathrm{HO} 1$ noted, causing the tool to be dealt with reluctance and skepticism. The fear of employee evaluation linked with salary reductions or even let-offs, especially during the financial crisis, resulted in a rather negative reaction of public servants towards the application, as $\mathrm{HO} 2$ mentioned. One can say, therefore, that one of the mistakes in the preparation of the application was the fact that it was introduced before employees were convinced for its usefulness, its practicality and its potential benefits.

As far as target setting is concerned, $\mathrm{HO}$ noted that many times the objectives set were vague, contradictory as well as conjectural. $\mathrm{HO} 2$ also added that in many cases they had a short-term character and were designed without any strategic planning.

HO3, moreover, notes that in implementing the procedure most employees stick to the adherence of the provisions of the Law rather than trying to achieve actual results. That caused a lack of focus on correctly applying the procedure as well as affected the quality of results, $\mathrm{HO} 1, \mathrm{HO}$ and LE2 mentioned.

One more critical point that caused MBO not to perform as expected in the Greek public sector was the fact, as $\mathrm{HO} 4$ and LE2 noted that the improvement proposals by DSOE, at the final stage of the implementation and before they reached the Ministerial office, were not forwarded. In essence, this means that the procedure was not concluded as it is provisioned and therefore it is difficult to expect improvement without proceeding with the improvement measures. Mistakes were also noted when the indicators were set, while sometimes, as $\mathrm{HO} 3$ 
and LE2 noted, they were not set at all. But even in cases where they were set they were either non-realistic or general in nature.

In connection with the above, after the first implementation of the MBO, DQE suggested specific measures in order to improve the implementation of the tool and its function. Unfortunately, as both HO1 and LE1 mentioned, these were not taken under consideration by higher administrative or political command.

Another important drawback of the implementation of MBO in the Greek public sector was the lack of time. Many employees complained that the time chosen for the processes of the tool to be performed was not convenient as it usually coincided with "rush-hours" of the offices. In addition, the procedures to be implemented constituted additional workload for everyone involved, and this further discredited the process in their eyes as $\mathrm{HO} 2$ and $\mathrm{HO} 3$ noted. Furthermore, Rammata mentions the large number of red-tape needed to start and conclude the application, while the absence of computerisation does not make procedures any easier to proceed.

Also, LE1 and LE2 mentioned the lack of administrative independence of the departments responsible for the $\mathrm{MBO}$, leaving no room for initiatives to the agencies while, at the same time, these agencies were left unprotected against political influence. A certain amount of administrative independence would allow public bureaus to perform better in their task to implement quality procedures. In addition, $\mathrm{HO} 3$ mentioned that vulnerability of $\mathrm{MBO}$ against external variables, such as economic, social and political which affected the outcomes of the application significantly.

As far as employees are concerned, $\mathrm{HO} 3, \mathrm{HO} 4$ and LE1 consider the lack of connection between employee productivity and salary, the lack of motives and incentives and the absence of rewards in general as one more disadvantage hindering the ideal MBO implementation. What was also pointed out was the absence of motives to seek further improvement once the application is completed, rather than just be satisfied with the results.

One of the important elements on which the success of the application depends upon is the participation of everyone involved in the procedure, regardless of their rank in the hierarchy. LE1 and LE2 noticed that, most of the times, this was not the case. The planning of the MBO procedure, by setting objectives and measuring indicators, was done almost exclusively by the managers. Similarly, another drawback is considered to be the lack of information of employees about the mission and vision of their organisation. In other words, 
employees don't have access to the "bigger picture" and this influenced their participation and their unwillingness to contribute in $\mathrm{MBO}$ procedures.

In regards to the positive impact of $\mathrm{MBO}$ use in Greek public administration these can be summarised as follows:

One of the most important positive impacts of MBO use was the enhancement of teamwork and the establishment of a culture of collaboration and trust between self-assessment team members and other employees and Departments. Good relationships between co-workers are of course always welcome in every organisation since the participation in a process of improvement made employees feel better about themselves, individually and as a part of a group with a common goal.

Objective setting contributes in the creation of a mentality of quality improvement. Every employee has its own targets to fulfill and that leads them to approach to try to achieve them more focused, rather than approach them like an everyday duty. This essentially makes employees use their abilities and expertise more effectively making them, ultimately, more productive.

Although the application of MBO faced many difficulties some public agencies, especially Regions and Municipalities, as HO1, HO6 and LE2, mentioned, claim to have seen improvement in their overall function, at least in some degree. Nevertheless, even in cases like this, it is difficult to display actual improvement in real terms, since any positive effects on the everyday function of the bureaus and society in general, except being difficult to measure, can hardly be identified.

\section{Concluding remarks: putting the pieces together}

The study which is presented in two separate papers analysed the implementation of two total quality tools, the CAF and the MBO, in Greek public administration, both of which are used widely in Europe and other countries as well, both in public as well as in private sector organisations. The implementation of the two applications can be beneficial in various ways for public services. Among other things, they can contribute to the improvement of the services provided and offer better customer service. They can also help in understanding how the agency is organised and operates, its strengths and weaknesses as well as in changing the attitude of employees towards the adoption of a quality culture.

The use of CAF and MBO by public services, despite their positive effects, was not without weaknesses and difficulties in their introduction and implementation in the Greek public sector. This influenced both the will of organisations to initiate and complete a self-assessment and/or goal setting as well as the quality and reliability of the final conclusions, thus preventing these 
tools to have the maximum positive impact in improving the public organisations which use them.

The most common difficulties of the application of the two tools have to do with:

- difficulties associated with the applications themselves, i.e. their structure and the proposed implementation procedures

- difficulties associated with the maturity level of the organisation to introduce and implement such organisational changes

- difficulties associated with the lack of commitment and support from leadership and the timing of application

- lack of information of the users, as well as the partalis-customers, on how these tools function and what the benefits from their use are.

What became immediately clear during the research, was that the public bodies in Greece were not ready for the introduction of principal management concepts. The lack of previous experience in TQM matters led to weaknesses and gaps in the preparation as well as their implementation.

What was missing, right from the start, was a framework of total quality policy, which could include both tools as part of a set of complementary actions with a joint targeting. In direct relation to the above, the preparatory measures implemented, as well as the time spent to introduce these applications were not sufficient enough to persuade the employees on their necessity, feasibility and their usefulness.

Also, the training of the staff was either patchy or, in many cases, nonexistent which resulted to the terminology used as well as the implementation procedure of the two applications (e.g. matching their content with the actual operation of the services) not being fully understood.

Moreover, in many cases the agencies did not set appropriate goals to address their weaknesses mainly because the CAF was not associated with addressing the specific problems through MBO. In other words it was not connected with addressing the essential problems of the services, but instead a general and vague application was made without substantial connection to the reality of the everyday function of the services. This resulted in limiting the potential improvements and benefits drastically.

Furthermore, regarding employees, it was ascertained that they have usually no knowledge of the overall operation of the General Directorate they belong to and thus do not see the "bigger picture" i.e. its mission and vision. In addition, another important disadvantage that was identified was the insufficient 
exchange of views and information within the organisation, as well as with other services using these two applications.

Public servants, being the operating arm of the decisions of political leadership, are most of the times not in the position to take any initiatives. In addition, the absence of a flexible organisational chart which would help avoiding additional workload is also considered a significant drawback.

One of the most important findings that the study is that although the implementation of the CAF helped in identifying the strengths and weaknesses of the services, this has not resulted in setting realistic goals through MBO. In other words, the diagnosis of the current function mode and organisational performance did not help to improve the quality of the action plans and that indicates lack of organic connection between self-assessment (CAF) and goalsetting (MBO). Conversely, but similarly, the application of goal setting requires the design of indicators in order for goals to be measurable - the latter, however, cannot be attainable unless based on strategic planning through the assessment of the function of the organisation which is done with the CAF, so, again, one tool needs, the other.

The low response rate to the found weaknesses, the low rate of implementation of the appropriate improvement actions and most importantly, the reasons this didn't happen (lack of time, other priorities, etc.), confirm the weaknesses for the effective integration of these applications to the operation of Greek public sector services.

Furthermore, in many cases there was no proper timing of the application which often coincided with the peak period of service hours thus further burdening workers. Both of these elements, as well as the additional paperwork the implementation of these tools requires, affected the subsequent attitude of the employees towards these applications.

Moreover, the benefits of the use of the two tools were not valued by their users as significant. This led to an overall, on their behalf, depreciation of the worth the applications as improvement tools and their actual response to the objectives they set. That can be explained by the inadequate entry and application process of the models, the non-implementation of the improvement measures (which devalues the efforts which were made up to this point), the lack of association between the two applications and, more generally, the lack of a comprehensive quality policy design in the public sector.

Directly related to the above is the interesting finding that, particularly in the case of CAF, its users believed that their participation in applying these tools did not particularly increase their interest in their service, most likely because they felt that their participation to the procedures was imposed, even though, at least in the case of CAF, this is optional. This fact consequently affected, their 
perception of the reasons the tools were implemented, the potential benefits from their use and the overall response of the model to the set targets.

Moreover, the diversity of conditions in which public services operate in our country prevents comparative learning and forbids them to capitalise from its benefits. The lack of basic common rules of operation for all services, such as a common way of recording the daily employee performance, does not help towards this direction.

In regards to the overall effect of the results of these applications on society, although sometimes visible, it is quite difficult to be measured in the absence of commonly agreed performance measuring indicators. No "social" performance indicators exist in order to establish the positive (or negative) effect of the qualitative actions of the public organisations in society.

Another important factor which should be emphasised is that despite the, gradual, introduction of modern technologies in Greek public services, most of the services still cannot be offered through the Internet. The great possibilities that technology offers, particularly via the Internet, can contribute greatly to the reduction and simplification of the required procedures until the completion of a request on behalf of the partalis.

The major benefit of the use of two applications referenced by, almost, everyone was that the application of the respective procedures created and embedded a collaborative environment and reinforced a sense of confidence among participants. The implementation of both tools was initially met with disbelief, nevertheless, through the contact circles of the self-evaluation and targeting groups, this hesitation was gradually eliminated and replaced by a feeling of satisfaction from participating in a report drafting and improvement process. However, the culture of teamwork, as a very important benefit, may be welcome, but in order to have real value for the services, it must present tangible benefits also.

Furthermore, the application of the CAF and MBO gave public services an incentive to focus, improve and simplify their processes by adopting new actions and setting new goals in order to upgrade their operations in the future. This way, additionally, the organisations strengthened their ability to take initiatives and develop strategies independently, no longer simply applying and following the directives of higher command. As mentioned earlier, some agencies claim to have seen improvement in their daily operation by the use of the two tools, but the lack of measurable results cannot prove these claims to be true in a convincing way.

Based on all of the above and taking into account the relevant factors cited, in order to increase the contribution of the tools under consideration in the 
improvement of the function of Greek public services, certain measures have been proposed.

Initially, there should be a comprehensive quality policy framework under which all agencies of the public sector will function by. Efforts should be made to adopt a quality culture, i.e. to create an environment where the values and principles of TQM will be part of the work mentality of all staff. To this goal Directorates should use modern human resources management approaches and not operate with outdated bureaucratic procedures. Under this spectrum the training of personnel in evaluation and quality procedures can prove to be vital.

Basic requirement, in any case, for the successful implementation of both tools, is the enduring support and encouragement of the organisation's political and administrative leadership towards their implementation. At the same time the staff must be supported and incentives provided for their further mobilisation and empowerment.

Moreover, leadership must create and disseminate a clear outline of the mission and vision of each General Directorate by setting specific targets, i.e. problems that we must addressed. Afterwards, the responsibilities of the employees must be shared according to their abilities and the nature of their work.

Furthermore, the adaptation of the tools to the peculiarities of the Greek public sector is of particular importance. There should be, in other words, further adjustment of the models to the reality and the particular characteristics (culture, structure, institutions, management strategy, etc.) of the Greek public administration. It is also necessary to further "popularise" these models. Simplification and clearer terminology in the instruction manuals of the provisioned procedures of the two tools is required in order for them to be understood by all involved in the process.

It is also necessary to enhance the belief of everyone involved in the procedure and note the importance of their contribution, thus more efforts to convince leaders and officials on the feasibility and usefulness of the use of both applications are required. Towards this TQM awareness and dissemination campaigns across the hierarchy, from Ministers and general Directors to employees of the lowest ranks, would be especially helpful.

Moreover, further institutional entrenchment of the two tools is necessary, which would add to their credibility and change the perception by employees towards their importance, however this should have a supporting character and not appear to be imposed. Furthermore, in the same direction, the reestablishment of the Quality Directorates and Departments in the Ministries, Regions and local Governments could also prove to be beneficial.

An even closer interconnection between CAF and MBO users would be crucial at a local, national and European level while, at the same time, "best 
practices" would be encouraged and promoted. The exchange of best practices facilitates comparative learning and disseminates them to other organisations as well. To this end educational seminars and quality conferences should be frequently organised and the participation of as much as possible organisations encouraged.

Another point which should be emphasised is that public agencies, in their effort to increase partali/users satisfaction, should be particularly interested in their comments and observations. Thus, the partali's opinion on what they consider to be "quality service" and if such is offered to them, should be taken under consideration. In this context, relevant surveys should be made at regular intervals, through which the, continuously, changing needs and requirements of the partalis can be, each time, revealed. Furthermore, informing the public on quality improvements in their operation and services provision could also prove to be very useful since it increases credibility.

Furthermore, the use of the two tools should be combined as well as complementary linked with other managerial tools (like flowcharts, control maps, a.o.) for better results. Of course, for this to happen relevant legislative provisions as well as the provision of information and the education of the users is required.

Nowadays, the introduction of modern technology in all government agencies is an absolute necessity. Partalis should not need to be visiting two or three different services and collect a large number of documents for the submission of their requests. The complete automation and the electronic interconnection between public services can contribute to this aim making them more efficient and effective.

In addition, the creation and use of electronic tools and programs specifically designed for the implementation of the CAF and MBO can simplify and support users in their task. In some EU countries such applications are already in use and were presented in quality conferences in the past.

Finally, the repetition of the applications is necessary, as this contributes to the creation of comparable information in relation to previous results, reducing the difficulties faced by their users and helps designing the appropriate improvement measures. In order for tools like the CAF and MBO to present results they must be applied continuously. At the same time, in order to analyses their results and recognise their usefulness, constant monitoring is necessary.

Despite the drawbacks and problems in their application, both the CAF and $\mathrm{MBO}$ are useful quality assessment and performance improvement tools which can be implemented by all public bodies, being, at the same time, flexible for modifications and adjustments. However, in order to maximise the benefits by their implementation and make proper use of their results, the improvement 
proposals must be put forward, a quality culture in the daily operation of the organisation be adopted, but above all the commitment and support of the political and administrative leadership ensured.

The findings of this study could be possibly used for further exploration of matters relative to the improvement of the quality of service of public bodies in Greece in the future. The research showed that the introduction of these tools in the services of the Greek public sector did not produce significant results. Many reasons were mentioned justifying this outcome, the most important being the lack of decisiveness of the political as well as the administrative leadership, to promote, encourage, and support these projects. The tools could prove to be useful and assist public services in improving their functions if they were implemented using the procedures that are provisioned by the Law, correctly and to their full extend. Unfortunately, this proved not to be the case in the agencies of the Greek public sector.

The quality of the state, i.e. in essence the quality of life partalis enjoy, is an indication of the quality of our democracy and so the modernisation of the public sector is a strategic challenge for the development of each country. Towards this, the incorporation of principal management concepts, such as the $\mathrm{CAF}$ and $\mathrm{MBO}$, can contribute to the achievement of the desired quality in services and improve the efficiency and effectiveness of public organisations. Naturally, this cannot be done automatically, since this kind of processes require effort, time, patience, determination but most of all a change in the mentality from everyone involved so the intended improvements are achieved in practice. 


\section{References}

[1] Borins, S. (1995). The new public management is here to stay. Canadian Public Administration, 38(1), pp. 122-132.

[2] Drucker, P. F. (1954). The practice of management. New York: Harper \& Row.

[3] Drucker, P. F. (1976). What Results Should You Expect ? A Users Guide to MBO. Public Administration Review, 36(1), pp. 12-19.

[4] EKDDA (National Centre for Public Administration and Local Government). (2012). Educational training at INEP (in Greek). Retrieved October 20, 2016, from http://www.ekdd.gr/ekdda/index.php/gr/2012-06-19-08-28-32

[5] Esmark, A. (2016). Maybe It Is Time to Rediscover Technocracy? An Old Framework for a New Analysis of Administrative Reforms in the Governance Era. Journal of Public Administration Research and Theory, 27(3), pp. 501-516.

[6] Hellenic Republic. Establishment of Management by Objectives, performance measurement, and other provisions (in Greek), Pub. L. No. 3230, Government Gazette 44/ A'/ 11.02.2004 (2004). Greece.

[7] Lee, G. (2011). Uncovering the blurring of sectors: A comparison of perceived organizational values between the public and nonprofit sectors. International Review of Public Administration, 16(3), pp. 1-23.

[8] Metaxas, I. N., \& Koulouriotis, D. E. (2014). A theoretical study of the relation between TQM, assessment and sustainable business excellence. Total Quality Management \& Business Excellence, 25(5-6), pp. 494-510.

[9] Pappas, T., \& Assimakopoulou, Z. (2012). Party Patronage in Greece: Political Entrepreneurship in a Party Patronage Democracy. In P. Kopecký, P. Mair, \& M. Spirova (Eds.), Party Patronage and Party Government in European Democracies (pp. 144-162). Oxford: Oxford University Press.

[10] Pollitt, C., \& Bouckaert, G. (2011). Public management reform: a comparative analysis. New public management, governance, and the neo-weberian state. Oxford: Oxford University Press.

[11] Psomas, E. L., \& Jaca, C. (2016). The impact of total quality management on service company performance: evidence from Spain. International Journal of Quality \& Reliability Management, 33(3), pp. 380-398.

[12] Rammata, M. (2011). Modern Greek Public Administration: Between Bureacracy and Management (in Greek). Athens: Kritiki.

[13] Rathgeb Smith, S. (2010). Nonprofits and Public Administration: Reconciling Performance Management and Partali Engagement. The American Review of Public Administration, 40(2), pp. 129-152.

[14] Staes, P., \& Thijs, N. (2005). Quality management on the European Agenda. EIPASCOPE, 1, 3341. Retrieved from http://www.eipa.eu/cms/repository/eipascope/scop05_1_6.pdf

[15] Staes, P., Thijs, N., Stoffels, A., \& Heidle, L. (2010). "10 years of CAF" - More than 2000 CAF users. EIPASCOPE, 2, 35-40. Retrieved from http://www.eipa.eu/files/repository/eipascope/20101022102451_Eipascope_2010_2_Repor t.pdf

[16] Tsekos, T. (2007). Public Service Quality and Public Marketing (in Greek). Athens: Ant. N. Sakkoulas Publications. 
HOLISTICA Vol 9, Issue 2, 2018

[17] Tsimboukidou, I., Dionysopoulou, P., \& Mylonakis, J. (2013). Total Quality Management Systems in the Hellenic Public Administration: A Case Study on the Common Assessment Framework Application. Public Administration Research, 2(2), pp. 124-133. 